\title{
Availability of Information on Reptile Health and Welfare from Stores Selling Reptiles
}

\author{
David L. Williams*, Rebecca Jackson \\ Queen's Veterinary School Hospital, Department of Veterinary Medicine, University of Cambridge, \\ Cambridge, England \\ Email: "dlw33@cam.ac.uk
}

Received 18 January 2016; accepted 14 March 2016; published 17 March 2016

Copyright (C) 2016 by authors and Scientific Research Publishing Inc.

This work is licensed under the Creative Commons Attribution International License (CC BY). http://creativecommons.org/licenses/by/4.0/

c) (i) Open Access

\begin{abstract}
The popularity of keeping reptiles such as snakes and lizards is ever increasing. The health and welfare of these animals depends on the knowledge and understanding of their environmental and nutritional needs. But where does a new owner of these species obtain such information? This study aims to investigate what information is available in pet shops and stores for the reptile owning public and how well this information is imparted. Our findings show that there is a lot of excellent advice on the husbandry of reptiles in some shops while others offer less advice. It is hoped that through this study areas of downfall on primary knowledge can be recognized so that all shops can aspire to the highest levels of information.
\end{abstract}

\section{Keywords}

Reptile, Health, Welfare, Care, Information

\section{Introduction}

It is widely recognized that for reptiles, as for all exotic companion animal species, husbandry is the key to health. And since reptiles are kept more and more widely as pets in the UK, we have to ask where new owners of snakes and lizards, chameleons and geckos are getting the information they need to ensure optimal husbandry for their new charges. In 2013 it was estimated that 400,000 lizards and 300,000 snakes were kept as household pets in the UK [1]. More than 30 years ago Cowan discussed the problem of maldapation where reptile health and well being was compromised by a number of failures in adequately providing for the physiological and be-

${ }^{*}$ Corresponding author. 
havioral needs of the animal [2]. It is widely recognized by those working with diseases of any captive exotic species that husbandry failures are key to providing for these animals in captivity. Yet we still see significant problems in reptile health and welfare because owners simply do not know how to care for their pets. Even in these times when the internet is a fount of information, pet shops that sell reptiles are often the primary source of information for those aiming to buy and keep these poikilothermic species, yet how well are those who work in such stores informed about the requirements, physiological and behavioural needs of the animals they are selling?

Warwick and colleagues have undertaken a survey of reptile and amphibian pet markets across the European Union with damning results [3] [4], yet their review does not detail ways in which the problems highlighted for reptile health and welfare can be overcome. In the present survey, it is hoped that, through evaluating the information available from the shops where these animals are sold, improvements can be made by optimizing such information and thus improving reptile health and welfare.

\section{Methods}

Fifty pet shops in several locations across the UK were visited over a 12 month period. Information on reptile care and welfare was gathered by asking those working in the store a structured set of questions. The questions particularly focused on species recommended for a first time reptile owner, recommended enclosure size and furnishings, requirements of the species involved regarding thermoregulation, humidity, ultraviolet illumination, feeding and nutrition, life expectancy, possible illnesses and availability of veterinary treatment.

\section{Results}

Of the 50 shops visited $33(66 \%)$ were general pet stores while 17 were specific reptile/exotic pet centres. $12 \%$ of the shops visited provided a specific member of staff to answer any questions related to the reptile species that they sold. Table 1 shows the results for all shops.

Table 2 demonstrates the species most commonly recommended by the store staff for first time reptile owners. The majority consisted of bearded dragons (Pogona vitticeps), leopard geckos(Eublepharis macularius) and corn snakes(Pantherophis guttatus).

Housing must promote the physical and psychological health and wellbeing of the reptile by allowing it to express natural behaviors, in particular locomotion, basking and hiding [5] [6]. 94\% of pet shops gave recommendations for vivarium size within the guideline limits previously set [7] [8] but with regard to advice on substrates to be used there were many and varying recommendations. The majority of shops (52\%) suggested bark or woodchips while $23 \%$ recommended sand. $5 \%$ recommended "reptile carpet" and $9 \%$ suggested kitchen roll or newspaper, whilst $4 \%$ recommended soil. $7 \%$ did not mention substrate. $70 \%$ of shops visited recommended providing a hiding place for the reptile. All pet shops which recommended purchasing an arboreal species recommended climbing apparatus. $4 \%$ of shops talked about "decor" suggesting that they may place more importance on the cosmetic appearance of the enclosure for the owner than for the requirements of the animal.

With regard to the key issue of temperature for these poikilothermic animals, shops made different recommendations for various species. $13 \%$ recommended the use of two sources of heat but only $8 \%$ discussed the importance of a temperature gradient. $22 \%$ of shops recommended a monitoring device be used to determine the temperature of the vivarium. Concerning snakes, $85 \%$ of shops suggested the use of a heat mat, whilst $15 \%$ recommended instead the use of an overhead heat source such as a ceramic bulb. With regard to lizards $60 \%$ of shops selling gecko species recommended the use of a heat mat only while $37 \%$ recommended the use of on overhead heating bulb. For bearded dragons (Pogona vitticeps), $83 \%$ recommended a ceramic heat bulb or a basking light. Humidity is central to optimal reptile husbandry, so it was thus concerning that few sellers mentioned humidity. Only $26 \%$ of shops visited gave any recommendations at all in verbal communication while $12 \%$ made no mention of humidity in the store but provided an information sheet which did give details of humidity requirements. The rest of the stores (62\%) provided no information. Similarly providing ultraviolet light is important in any species of reptile. With regard to snakes, no sellers made any recommendation regarding the purchase of an ultraviolet source while $80 \%$ of stores offering bearded dragon for sale recommended ultraviolet light use. Only $35 \%$ of those offering leopard geckos for sale advised purchase of an ultraviolet light source. No discussion was made by any seller about the benefits or harm of ultraviolet, nor the intensity of bulbs to use or the need to replace the bulb after 6 months. 
Table 1. Data recorded from 50 pet shops and reptile stores.

\begin{tabular}{|c|c|c|c|c|c|c|c|c|c|c|c|c|}
\hline Store & Type & Size & Species & $\begin{array}{c}\text { Enclosure } \\
\text { Size }\end{array}$ & Substrate & Furniture & Temperature & Humidity & $\mathbf{U V}$ & $\begin{array}{c}\text { Life } \\
\text { Expectancy }\end{array}$ & Vet & Diet \\
\hline 1 & GPS & $\mathrm{S}$ & Any & NS & $\mathrm{S}$ & NM & HM for CS & $\mathrm{Y}$ & $\mathrm{N}$ & CS-10y & RS & $\begin{array}{l}\mathrm{L} \text { for } \mathrm{LG} \text { every other } \\
\text { day + bowl of Ca once } \\
\text { a week; R for CS } 3-4 \\
\text { d for young, once } \\
\text { a week for older }\end{array}$ \\
\hline 2 & GPS & $\mathrm{S}$ & $\mathrm{CS}$ & $3 \mathrm{ft}$ viv & B & $\mathrm{H}$ & $\mathrm{HM}, \mathrm{M}, \mathrm{G}$ & $\mathrm{Y}$ & $\mathrm{N}$ & CS-20y & NK & $\begin{array}{l}\text { R } 3 \text { - } 5 \text { days, put in } \\
\text { small dark box; } \\
\text { don't touch for } \\
3 \text { - } 4 \text { days after or } \\
\text { will regurgitate }\end{array}$ \\
\hline 3 & RS & $\mathrm{L}$ & $\begin{array}{l}\mathrm{LG}, \mathrm{BD}, \\
\mathrm{CS}\end{array}$ & $3 \mathrm{ft}$ viv & B & $\mathrm{H}$ & $\begin{array}{l}\text { HB for BD, } \\
\text { LG. HM } \\
\text { for CS }\end{array}$ & NM & $\mathrm{N}$ & $\begin{array}{l}\text { LG, } \\
\text { BD-9-15y, } \\
\text { CS-20y }\end{array}$ & RS & $\begin{array}{l}\text { LG and BG-L } \\
\text { one day, S the next; } \\
\text { CS - R once a week }\end{array}$ \\
\hline 4 & RS & $\mathrm{L}$ & Any & $\begin{array}{l}3 \mathrm{ft} \text { for lizards; } \\
\mathrm{CS} \text { increasing } \\
\text { to } 4 \mathrm{ft} \text { as grows }\end{array}$ & KR & $\mathrm{H}$ & Dep on spp & $\mathrm{Y}$ & $\mathrm{Y}$ & $\begin{array}{l}\text { BD, } \\
\text { LG-15-20y; } \\
\text { CS-20y }\end{array}$ & RS & $\begin{array}{l}\text { Lizards-L every } \\
\text { other day, S ED; } \\
\text { CS-R once a week }\end{array}$ \\
\hline 5 & RS & $\mathrm{L}$ & $\begin{array}{l}\text { LG, BD, } \\
\text { CS }\end{array}$ & $\begin{array}{l}\mathrm{LG} \text { and } \\
\mathrm{CS}-2 \mathrm{ft} \\
\mathrm{BD}-4 \mathrm{ft}\end{array}$ & $\mathrm{S}$ & $\mathrm{H}$ & $\begin{array}{l}\text { HM for } L G \text {, } \\
C S . H B \text { and } \\
M \text { for BD }\end{array}$ & $\mathrm{Y}$ & $\mathrm{N}$ & BD-20y & $\mathrm{RS}$ & $\begin{array}{l}\text { LG-L ED; } \\
\text { BD-L and S ED; } \\
\text { CS frozen R } \\
\text { once a week }\end{array}$ \\
\hline 6 & GPS & $\mathrm{S}$ & $\mathrm{BD}$ & $\begin{array}{l}2 \mathrm{ft} \text { when young, } \\
3 \mathrm{ft} \text { when fully } \\
\text { grown }\end{array}$ & B & $\mathrm{H}$ & $\mathrm{B}, \mathrm{M}, \mathrm{G}$ & $\mathrm{Y}$ & $\mathrm{N}$ & NK & PS & $\mathrm{L}$ and $\mathrm{S} E D$ \\
\hline 7 & GPS & $\mathrm{L}$ & Any & $\begin{array}{l}\text { Small viv then } \\
\text { bigger by age }\end{array}$ & $\mathrm{S}, \mathrm{B}$ & $\mathrm{H}, \mathrm{C}$ & $\begin{array}{l}\text { HM for CS. } \\
\text { HM and } M \\
\text { for } L G, B D\end{array}$ & $\mathrm{Y}$ & $\mathrm{Y}$ & $\begin{array}{l}\text { LG-25y; } \\
\text { BD-8-10y; } \\
\text { CS-15y }\end{array}$ & RS & $\begin{array}{l}\text { Lizards-L every } \\
\text { other day, S ED; } \\
\text { CS-once a week R } \\
\text { increasing in size } \\
\text { as grow }\end{array}$ \\
\hline 8 & GPS & $\mathrm{S}$ & $\mathrm{BD}, \mathrm{CS}$ & $\begin{array}{l}3 \mathrm{ft} \text { viv for BD; } \\
\mathrm{CS} \text { depends } \\
\text { on size }\end{array}$ & B & $\mathrm{H}$ & $\begin{array}{l}\mathrm{HM} \text { for } \mathrm{CS} \text {. } \\
\mathrm{B} \text { for } \mathrm{BD}\end{array}$ & $\mathrm{Y}$ & $\mathrm{Y}$ & BD-15y & PS & $\begin{array}{l}\text { BD-S and S ED; } \\
\text { CS-frozen R } \\
\text { once a week }\end{array}$ \\
\hline 10 & GPS & $\mathrm{S}$ & $\mathrm{BD}$ & $3 \mathrm{ft}$ viv & NM & NM & HM & NM & $\mathrm{Y}$ & BD-16y & Any & $\mathrm{S}$ and $\mathrm{L}$ ED \\
\hline 11 & RS & $\mathrm{L}$ & $\begin{array}{l}\mathrm{LG}, \mathrm{BD}, \\
\mathrm{CS}\end{array}$ & $\begin{array}{l}\text { LG-1ft viv; } \\
\text { BD-3ft viv; } \\
\text { CS-small to } \\
\text { 1-3ft as grow }\end{array}$ & B & $\mathrm{H}$ & $\begin{array}{l}\mathrm{HM} \text { for } \mathrm{LG}, \\
\mathrm{CS} . \mathrm{B} \text { for } \mathrm{BD} \text {. } \\
\mathrm{G} \text { and } \mathrm{M}\end{array}$ & NM & Y & $\begin{array}{l}\text { LG-8y; } \\
\text { BD-10y; } \\
\text { CS-15y }\end{array}$ & $\mathrm{RS}$ & $\begin{array}{l}\text { Lizards-L ED; } \\
\text { CS-R once a week } \\
\text { - do not handle day } \\
\text { after feed or will } \\
\text { regurgitate. Ca also }\end{array}$ \\
\hline 12 & RS & $\mathrm{L}$ & $\mathrm{BD}, \mathrm{LG}$ & BD-3ft viv & $\mathrm{S}$ & C, B & HM for $L G$ & NM & $\begin{array}{l}\text { Y for } \\
\text { diurnal } \\
\text { spp }\end{array}$ & $\begin{array}{l}\text { BD, } \\
\text { LG-14-16y }\end{array}$ & RS & $\begin{array}{l}\text { LG-L ED; BD-L } \\
\text { and S ED and then } \\
\text { every other day as } \\
\text { get older }\end{array}$ \\
\hline 13 & GPS & M & $\mathrm{BD}, \mathrm{CS}$ & $\begin{array}{l}\text { Lizards-3ft viv; } \\
\text { Snakes-smaller } \\
\text { to larger viv }\end{array}$ & $\mathrm{S}$ & $\mathrm{H}$ & $\begin{array}{l}\mathrm{HM} \text { for } \mathrm{CS} \text {. } \\
\mathrm{HB} \text { for } \mathrm{BD} \text {, } \\
\mathrm{LG}\end{array}$ & NM & $\mathrm{Y}$ for $\mathrm{BD}$ & BD-10y & RS & $\begin{array}{l}\text { CS-R dep on size } \\
\text { once a week; LG-L } \\
\text { ED; BD-S ED and } \\
6 \text { crickets once } \\
\text { a week }\end{array}$ \\
\hline 14 & RS & M & $\mathrm{BD}$ & $4 \mathrm{ft}$ viv for BD & NM & $\mathrm{D}$ & HB & $\mathrm{HO}$ & $\mathrm{N}$ & $\begin{array}{l}\text { BD-10y. } \\
\text { CS-20-25y }\end{array}$ & $\mathrm{RS}$ & L ED \\
\hline 15 & GPS & M & $\mathrm{BD}$ & $3 \mathrm{ft}$ viv & $\mathrm{KR}$ & $\mathrm{H}$ & HB & NM & $\mathrm{Y}$ for $\mathrm{BD}$ & BD-15y & NK & $\mathrm{L}$ and $\mathrm{S}$ ED \\
\hline 16 & GPS & M & $\begin{array}{l}\mathrm{LG}, \mathrm{BD}, \\
\mathrm{CS}\end{array}$ & $\begin{array}{l}\text { BD-3ft viv; } \\
\text { LG-2ft viv }\end{array}$ & $\mathrm{S}$ & $\mathrm{H}$ & $\begin{array}{l}\mathrm{HM} \text { for } \mathrm{CS} \text {. } \\
\mathrm{HB} \text { for } \mathrm{BD} \text {, } \\
\mathrm{LG}\end{array}$ & NM & Y for BD & $\begin{array}{l}\text { BD, } \\
\text { LG-15y. } \\
\text { CS-20y }\end{array}$ & $\mathrm{RS}$ & $\begin{array}{l}\text { CS-R dep on size } \\
\text { every } 4 d \text { when young, } \\
\text { every wk when older; } \\
\text { LG-L ED. BDs-S } \\
\text { ED and L every } \\
\text { other day }\end{array}$ \\
\hline 17 & GPS & M & $\begin{array}{l}\mathrm{LG}, \mathrm{BD} \text {, } \\
\mathrm{CS}\end{array}$ & $\begin{array}{l}\text { LG-2ft viv; } \\
\text { BD-4ft viv; } \\
\text { CS-3ft viv }\end{array}$ & $\mathrm{B}, \mathrm{KR}$ & $\mathrm{H}$ & $\begin{array}{l}\mathrm{HM} \text { for } \mathrm{CS} \text {. } \\
\mathrm{HB} \text { for } \mathrm{LG} \text {, } \\
\mathrm{BD}\end{array}$ & NM & Y for BD & $\begin{array}{l}\text { LBD, } \\
\text { LG-10y. } \\
\text { CS-20y }\end{array}$ & Any & $\begin{array}{l}\text { BD-S ED and } \\
\text { L ED when young; } \\
\text { CS R once a week }\end{array}$ \\
\hline
\end{tabular}




\begin{tabular}{|c|c|c|c|c|c|c|c|c|c|c|c|c|}
\hline \multicolumn{13}{|c|}{ Continued } \\
\hline 18 & GPS & M & $\mathrm{BD}$ & $3 \mathrm{ft}$ viv & $\mathrm{B}$ & NM & $\mathrm{HB}$ and $\mathrm{M}$ & NM & $\mathrm{Y}$ & BD-7-10y & Any & L ED \\
\hline 19 & GPS & M & $\mathrm{BD}, \mathrm{LG}$ & $3 \mathrm{ft}$ viv & B & $\mathrm{H}$ & $\begin{array}{l}\text { HB for BD. } \\
\text { HM for } L G\end{array}$ & NM & $\begin{array}{l}\text { Y for BD, } \\
\text { if want } \\
\text { for } L G\end{array}$ & BD-7-10y & $\mathrm{RS}$ & $\mathrm{L}$ and $\mathrm{S}$ ED \\
\hline 20 & RS & M & $\mathrm{BD}$ & $\begin{array}{l}\text { BD and CS- } \\
3 \mathrm{ft} \text { viv; LG- } \\
\text { smaller viv }\end{array}$ & $\mathrm{KR}$ & $\mathrm{H}$ & $\begin{array}{l}\text { HB for BD, } \\
\mathrm{LG}, \mathrm{CS}\end{array}$ & NM & $\mathrm{Y}$ for $\mathrm{BD}$ & BD-10y & NK & $\begin{array}{l}\text { BD-S and L every } \\
\text { other day; LG-L ED; } \\
\text { CS-R once a week }\end{array}$ \\
\hline 21 & GPS & M & $\mathrm{LG}, \mathrm{BD}, \mathrm{CS}$ & $\begin{array}{l}\text { BD and } \\
\text { CS-3ft viv; } \\
\text { smaller } \\
\text { for LGs }\end{array}$ & B & $\mathrm{H}$ & $\begin{array}{l}\text { HM for } L G \text {, } \\
\text { CS. HB for } \\
\text { BG. }\end{array}$ & NM & $\mathrm{Y}$ for $\mathrm{BD}$ & BD-10y & NK & $\begin{array}{l}\text { BD-S plus L ED when } \\
\text { young and every other } \\
\text { day when older; LG-L } \\
\text { only every day; CS-R } \\
\text { once a week }\end{array}$ \\
\hline 22 & GPS & $\mathrm{S}$ & $\mathrm{BD}, \mathrm{LG}$ & $\begin{array}{l}\mathrm{BD}, \mathrm{CS}-3-4 \mathrm{ft} \\
\text { viv; LG-2ft viv }\end{array}$ & $\mathrm{S}$ & $\mathrm{H}$ & $\begin{array}{l}\text { HM and } G \\
\text { for } L G, C S \text {. } \\
\text { HB for BD }\end{array}$ & NM & $\mathrm{Y}$ for BD & BD-7-10y & Any & $\begin{array}{l}\text { Geckos-L ED; BD-L and } \\
\text { S on alternative days; } \\
\text { CS-R once a week }\end{array}$ \\
\hline 23 & RS & $\mathrm{L}$ & $\begin{array}{l}\mathrm{LG}, \mathrm{CG} \\
\mathrm{BD}, \mathrm{CS}\end{array}$ & $\begin{array}{l}\text { BD-2-4ft viv; } \\
\text { LG-2ft viv; } \\
\text { CG-tall viv }\end{array}$ & So & $\mathrm{H}$ & $\begin{array}{l}\text { HM for CS, } \\
\text { LG. Ba for } \\
\text { BD }\end{array}$ & NM & $\mathrm{Y}$ for BD & $\begin{array}{l}\text { BD-10-15y. } \\
\text { CS, LG-20y }\end{array}$ & PS & $\begin{array}{l}\text { LG-L ED; BD-S ED } \\
\text { and L every other day; } \\
\text { CS-R once a week }\end{array}$ \\
\hline 24 & GPS & $\mathrm{L}$ & $\begin{array}{l}\mathrm{LG}, \mathrm{BD}, \\
\mathrm{CS}\end{array}$ & $\begin{array}{l}\text { LG-2-3ft viv; } \\
\text { BD-4ft viv; } \\
\text { CS-2-4ft viv }\end{array}$ & $\mathrm{B}, \mathrm{C}$ & $\mathrm{H}$ & $\begin{array}{l}\text { HM for } L G, \\
\text { CS. HB for } \\
\text { BD }\end{array}$ & $\mathrm{HO}$ & $\mathrm{Y}$ for BD & $\begin{array}{l}\text { BD-10-15y; } \\
\text { LG-7-10y }\end{array}$ & RS & $\begin{array}{l}\text { LG-L ED when young } \\
\text { and decrease as get } \\
\text { older; CS-R once } \\
\text { a week; BD-as LGs } \\
\text { plus S }\end{array}$ \\
\hline 25 & GPS & $\mathrm{M}$ & $\begin{array}{l}\mathrm{BD}, \mathrm{CG}, \\
\mathrm{CS}\end{array}$ & $\begin{array}{l}\text { BD-min of } \\
3 \mathrm{ft} \text { viv; CG, } \\
\text { CS-tall viv }\end{array}$ & So, B & $\mathrm{C}, \mathrm{H}$ & $\begin{array}{l}\mathrm{HM} \text { for } \mathrm{CS} \text {. } \\
\mathrm{HB} \text { for } \mathrm{BD}\end{array}$ & $\mathrm{Y}$ & $\mathrm{Y}$ for $\mathrm{BD}$ & BD-10-15y & $\mathrm{RS}$ & $\begin{array}{l}\text { CG-dry powder diet } \\
\text { ED; BD-S and L on } \\
\text { alternative days; } \\
\text { CS-R once a week }\end{array}$ \\
\hline 26 & GPS & $\mathrm{S}$ & $\mathrm{BD}, \mathrm{LG}$ & $3-4 \mathrm{ft}$ viv & $\mathrm{B}$ & $\mathrm{H}$ & HB & NM & $\begin{array}{l}\text { Y for BD, } \\
\text { if want } \\
\text { for } \mathrm{LG}\end{array}$ & BD-15y & $\mathrm{RS}$ & $\begin{array}{l}\text { LG-L ED; } \\
\text { BDs-S and L ED }\end{array}$ \\
\hline 27 & GPS & $\mathrm{S}$ & $\mathrm{BD}, \mathrm{LG}$ & $\begin{array}{l}\text { BD-4ft viv; } \\
\text { LG-2-3ft viv }\end{array}$ & B & $\mathrm{H}$ & $\begin{array}{l}\text { HB for BD. } \\
\mathrm{HB} \text { or } \mathrm{HM} \\
\text { for } \mathrm{LG}\end{array}$ & NM & $\mathrm{Y}$ & $\begin{array}{l}\text { BD-10y; } \\
\text { LG-7-10y }\end{array}$ & RS & $\begin{array}{l}\text { LG-L ED when young, } \\
3 \text { - } 4 \text { times a week } \\
\text { when older; BDs } \\
\text { L every other d }\end{array}$ \\
\hline 28 & GPS & M & $\mathrm{BD}, \mathrm{CS}$ & $3-4 \mathrm{ft}$ viv for all & B & $\mathrm{H}$ & $\mathrm{HB}$ and $\mathrm{M}$ & $\mathrm{Y}$ & $\mathrm{Y}$ & $\begin{array}{l}\text { BD-10; } \\
\text { CS-20y }\end{array}$ & NK & $\begin{array}{l}\text { BDs-S plus L ED when } \\
\text { young, decrease as get } \\
\text { older. Dust with } \mathrm{Ca}\end{array}$ \\
\hline 29 & GPS & $\mathrm{S}$ & $\mathrm{BD}, \mathrm{LG}$ & $\begin{array}{l}\text { LG and } \\
\text { CS-3ft viv }\end{array}$ & $\mathrm{B}, \mathrm{KR}$ & NM & $\mathrm{HM}$ & $\mathrm{Y}$ & $\mathrm{N}$ & $\begin{array}{l}\text { LG-10y; } \\
\text { CS-15-20y }\end{array}$ & NK & $\begin{array}{l}\text { LG-L ED; CS-R once } \\
\text { a week }\end{array}$ \\
\hline 30 & RS & $\mathrm{M}$ & $\begin{array}{l}\mathrm{LG}, \mathrm{BD}, \\
\mathrm{CS}\end{array}$ & $\begin{array}{l}\text { BD-4ft viv; } \\
\text { LG-2-3ft viv }\end{array}$ & B & $\mathrm{H}$ & $\begin{array}{l}\text { HB for BD. } \\
\text { HM for CS, } \\
\text { LG }\end{array}$ & NM & $\mathrm{Y}$ for $\mathrm{BD}$ & $\begin{array}{l}\text { BD, } \\
\text { LG-10-15y; } \\
\text { CS-15-20y }\end{array}$ & $\mathrm{RS}$ & $\begin{array}{l}\text { BD-S and L on } \\
\text { alternative days; } \\
\text { LG-L ED; CS-R every } \\
5 \mathrm{~d} \text { when young to } \\
\text { every week when older }\end{array}$ \\
\hline 31 & GPS & $\mathrm{S}$ & $\mathrm{BD}$ & $3-4 \mathrm{ft}$ & B & $\mathrm{H}$ & HB & NM & $\mathrm{Y}$ & BD-17y & NK & $\mathrm{L}$ and $\mathrm{S} \mathrm{ED}$ \\
\hline 32 & GPS & M & $\mathrm{BD}, \mathrm{CS}$ & $\begin{array}{l}\text { BD and } \\
\text { CS-3-4ft viv }\end{array}$ & B & $\mathrm{H}$ & $\begin{array}{l}\mathrm{HB} \text { for } \mathrm{BD} \text {, } \\
\mathrm{CS}\end{array}$ & $\mathrm{NM}$ & $\mathrm{Y}$ for $\mathrm{BD}$ & BD-15y & Any & $\begin{array}{l}\text { CS-R once a week; } \\
\text { BD-S and L 50:50 ED, } \\
\text { decrease to } 80: 20 \text { as } \\
\text { get older }\end{array}$ \\
\hline 33 & GPS & $\mathrm{S}$ & $\mathrm{BD}, \mathrm{RP}$ & $\begin{array}{l}\text { BD-3ft viv; } \\
\text { RP-2ft viv }\end{array}$ & B & $\mathrm{H}$ & $\begin{array}{l}\mathrm{HM} \text { for } \mathrm{CS} . \\
\mathrm{Ba} \text { for } \mathrm{BD}\end{array}$ & $\mathrm{HO}$ & $\mathrm{Y}$ for BD & NK & $\mathrm{RS}$ & $\begin{array}{l}\text { BD-S and L on } \\
\text { alternative days; } \\
\text { CS-R once a week }\end{array}$ \\
\hline 34 & GPS & $\mathrm{S}$ & $\mathrm{BD}$ & 3-4 ftviv & B & $\mathrm{H}$ & HB & $\mathrm{NM}$ & $\mathrm{Y}$ & NK & NK & $\mathrm{S}$ and $\mathrm{L}$ ED \\
\hline 35 & GPS & $\mathrm{S}$ & $\mathrm{BD}$ & $3-4 \mathrm{ft}$ viv & B & $\mathrm{H}$ & $\mathrm{B}, \mathrm{G}, \mathrm{M}$ & NM & $\mathrm{Y}$ & BD-15y & $\mathrm{RS}$ & $\mathrm{S}$ and $\mathrm{L} \mathrm{ED}$ \\
\hline 36 & GPS & $\mathrm{S}$ & $\mathrm{LG}, \mathrm{BD}$ & $\begin{array}{l}\text { BD-3-4ft viv; } \\
\text { LG-2ft high viv }\end{array}$ & B & $\mathrm{H}$ & $\mathrm{HB}$ for $\mathrm{BD}$ & NM & $\mathrm{N}$ & BD-9-10y & $\mathrm{RS}$ & $\begin{array}{l}\text { L every other day; } \\
\text { S ED for BD }\end{array}$ \\
\hline 37 & GPS & $\mathrm{S}$ & LG, BD, CS & $\begin{array}{l}\text { BD-3-4ft viv; } \\
\text { Gecko, } \\
\text { CS-2ft viv }\end{array}$ & B & $\mathrm{H}$ & $\begin{array}{l}\text { HB and M } \\
\text { for BD. HB } \\
\text { for CS. HM } \\
\text { for } L G\end{array}$ & NM & $\mathrm{Y}$ for BD & BD-15y & $\mathrm{RS}$ & $\begin{array}{l}\text { BD-S and L every } \\
\text { other day; LG-L } \\
\text { every other day; } \\
\text { CS-R every week }\end{array}$ \\
\hline 38 & RS & $\mathrm{L}$ & $\mathrm{BD}$ & $3 \mathrm{ft}$ viv & B & $\mathrm{H}$ & HB & NM & $\mathrm{Y}$ for BD & BD-15y & $\mathrm{RS}$ & $\begin{array}{l}\mathrm{L} \text { on alternative } \mathrm{d} \\
\text { and any type of } \\
\text { non-citrus fruits }\end{array}$ \\
\hline
\end{tabular}




\section{Continued}

\begin{tabular}{|c|c|c|c|c|c|c|c|c|c|c|c|c|}
\hline 39 & GPS & $\mathrm{S}$ & LG & $\begin{array}{l}\text { NS. Snakes in } \\
\text { viv plus half } \\
\text { length of snake }\end{array}$ & $\mathrm{C}$ & $\mathrm{H}$ & $\begin{array}{l}\mathrm{HM} \text { and } \mathrm{M} \\
\text { for } \mathrm{LG}\end{array}$ & $\mathrm{HO}$ & If wanted & LG-20y & Any & $\begin{array}{l}\text { LG-L ED; } \\
\text { BD } 80 \% \text { S/20\% L. } \\
\text { CS-R }\end{array}$ \\
\hline 40 & GPS & $\mathrm{L}$ & LG & $\begin{array}{l}4 \mathrm{ft} \text { viv plus } \\
\text { vertical for } \\
\text { some geckos. } \\
\text { CS-3ft }\end{array}$ & $\mathrm{B}$ & $\mathrm{H}, \mathrm{C}$ & $\begin{array}{l}\text { HB for } \mathrm{LG} \text {. } \\
\mathrm{HM} \text { for } \mathrm{CS}\end{array}$ & NM & $\mathrm{Y}$ & BD-10y & RS & $\begin{array}{l}\text { L ED. Geckos - baby } \\
\text { fruit food, } L \text { with } \\
\text { vitamin dusting. CS - R }\end{array}$ \\
\hline 41 & GPS & $\mathrm{S}$ & $\mathrm{BD}$ & $90 \mathrm{~cm}$ viv & $\mathrm{S}$ & $\mathrm{D}$ & $\mathrm{B}, \mathrm{M}$ & $\mathrm{HO}$ & $\mathrm{Y}$ for $\mathrm{BD}$ & BD-10y & PS & $\begin{array}{l}\mathrm{L} \text { and } \mathrm{S} \text { ED. Ca and } \\
\text { vitamin supplements }\end{array}$ \\
\hline 42 & RS & $\mathrm{L}$ & $\mathrm{LG}, \mathrm{BD}$ & $\begin{array}{l}\mathrm{BD}-3 \times 2 \times 2 \\
\text { viv. } \mathrm{LG}-2 \mathrm{ft} \text { min }\end{array}$ & NM & $\mathrm{H}$ & HB & NM & $\mathrm{Y}$ for $\mathrm{BD}$ & $\begin{array}{l}\text { BD-10-12y; } \\
\text { LG-20y }\end{array}$ & PS & $\begin{array}{l}\mathrm{L} \text {, plus } \mathrm{S} \text { and occas } \mathrm{R} \\
\text { for BD. Ca supplement }\end{array}$ \\
\hline 43 & RS & M & LG, BD & $\begin{array}{l}\text { BD }-4 \times 2 \times 2 \\
\text { ftviv. } L G-2 \\
\times 3 \text { ftviv }\end{array}$ & $\mathrm{S}$ & $\mathrm{NM}$ & $\begin{array}{l}\text { B for BD. } \\
\text { HM for } L G\end{array}$ & NM & $\mathrm{Y}$ for $\mathrm{BD}$ & $\begin{array}{l}\text { BD-8-10y; } \\
\text { LG-15y }\end{array}$ & RS & $\mathrm{L}$ plus $\mathrm{S}$ for $\mathrm{BD}$ \\
\hline 44 & RS & $\mathrm{L}$ & $\begin{array}{l}\mathrm{LG}, \mathrm{BD} \text {, } \\
\mathrm{TR}\end{array}$ & $\begin{array}{l}3 \mathrm{ft} \text { viv. } \\
\text { Taller for CGs }\end{array}$ & NM & NM & HM, M & NM & $\mathrm{Y}$ & BD-8y & RS & $\begin{array}{l}\mathrm{L} \text { for all lizards plus } \\
\mathrm{S} \text { for } \mathrm{BD}\end{array}$ \\
\hline 45 & RS & M & $\mathrm{BD}, \mathrm{LG}$ & $3 \mathrm{ft}$ viv & S, B & $\mathrm{H}$ & $\mathrm{HM}$ & NM & $\mathrm{N}$ & $\begin{array}{l}\text { BD-7-10y; } \\
\text { CS-20y }\end{array}$ & NK & $\begin{array}{l}\text { BD-S plus L. CS-R } \\
\text { dep on size }\end{array}$ \\
\hline 46 & RS & M & $\mathrm{BD}, \mathrm{LG}$ & $3 \mathrm{ft}$ viv & $\mathrm{S}$ & NM & $\begin{array}{l}\mathrm{HM} \text { for } \mathrm{LG} \text {. } \\
\mathrm{Ba} \text { for } \mathrm{BD}\end{array}$ & NM & $\mathrm{Y}$ for $\mathrm{BD}$ & $\begin{array}{l}\text { BD-15y; } \\
\text { LG-10y }\end{array}$ & RS & $\mathrm{L}$ plus $\mathrm{S}$ for $\mathrm{BD}$ \\
\hline 47 & GPS & $\mathrm{S}$ & $\begin{array}{l}\mathrm{LG}, \mathrm{BD}, \\
\mathrm{CS}\end{array}$ & $\begin{array}{l}3 \mathrm{ft} \text { viv. } \mathrm{CG} \\
\text { needs taller } \\
(2 \mathrm{ft} \text { high) }\end{array}$ & $\mathrm{C}$ & $\mathrm{H}$ & $\mathrm{HM}$ & $\mathrm{HO}$ & $\mathrm{N}$ & NK & RS & $\begin{array}{l}\text { CG-fruit puree; } \\
\text { LG L. BD-20\% } \\
\text { S, } 80 \% \text { L }\end{array}$ \\
\hline 48 & RS & $\mathrm{L}$ & Any & $\begin{array}{l}\text { LG- } 2 \mathrm{ft} \text { high } \\
\text { viv. BD changes } \\
\text { with growth }\end{array}$ & B & $\mathrm{H}, \mathrm{C}$ & $\begin{array}{l}\mathrm{HM} \text { for } \mathrm{LG} \text {. } \\
\mathrm{Ba} \text { for } \mathrm{BD}\end{array}$ & NM & $\mathrm{Y}$ for $\mathrm{BD}$ & $\begin{array}{l}\text { LG-18-22y; } \\
\text { BD-8-10y }\end{array}$ & NK & $\begin{array}{l}\text { LG-L. BD - } 20 \% \text { live, } \\
80 \% \text { veg }\end{array}$ \\
\hline 49 & RS & $\mathrm{L}$ & Any & NS & NM & $\mathrm{H}, \mathrm{C}$ & $\mathrm{Ba}$ & NM & $\mathrm{Y}$ & $\begin{array}{l}\text { BD-10-15y; } \\
\text { LG-20y; } \\
\text { CS-20-25y }\end{array}$ & RS & $\begin{array}{l}\text { LG: L and occas R, } \\
\text { Ca in bowl. } \\
\text { BD: L AND S. } \\
\text { CS: R dep on size }\end{array}$ \\
\hline 50 & GPS & $\mathrm{S}$ & $\mathrm{BD}$ & $3 \mathrm{ft}$ viv & $\mathrm{S}$ & $\mathrm{H}$ & $\mathrm{Ba}, \mathrm{G}$ & NM & $\mathrm{Y}$ for $\mathrm{BD}$ & BD-10y & RS & $25 \% \mathrm{~S}, 75 \% \mathrm{~L}$ \\
\hline
\end{tabular}

Codes for Table 1.

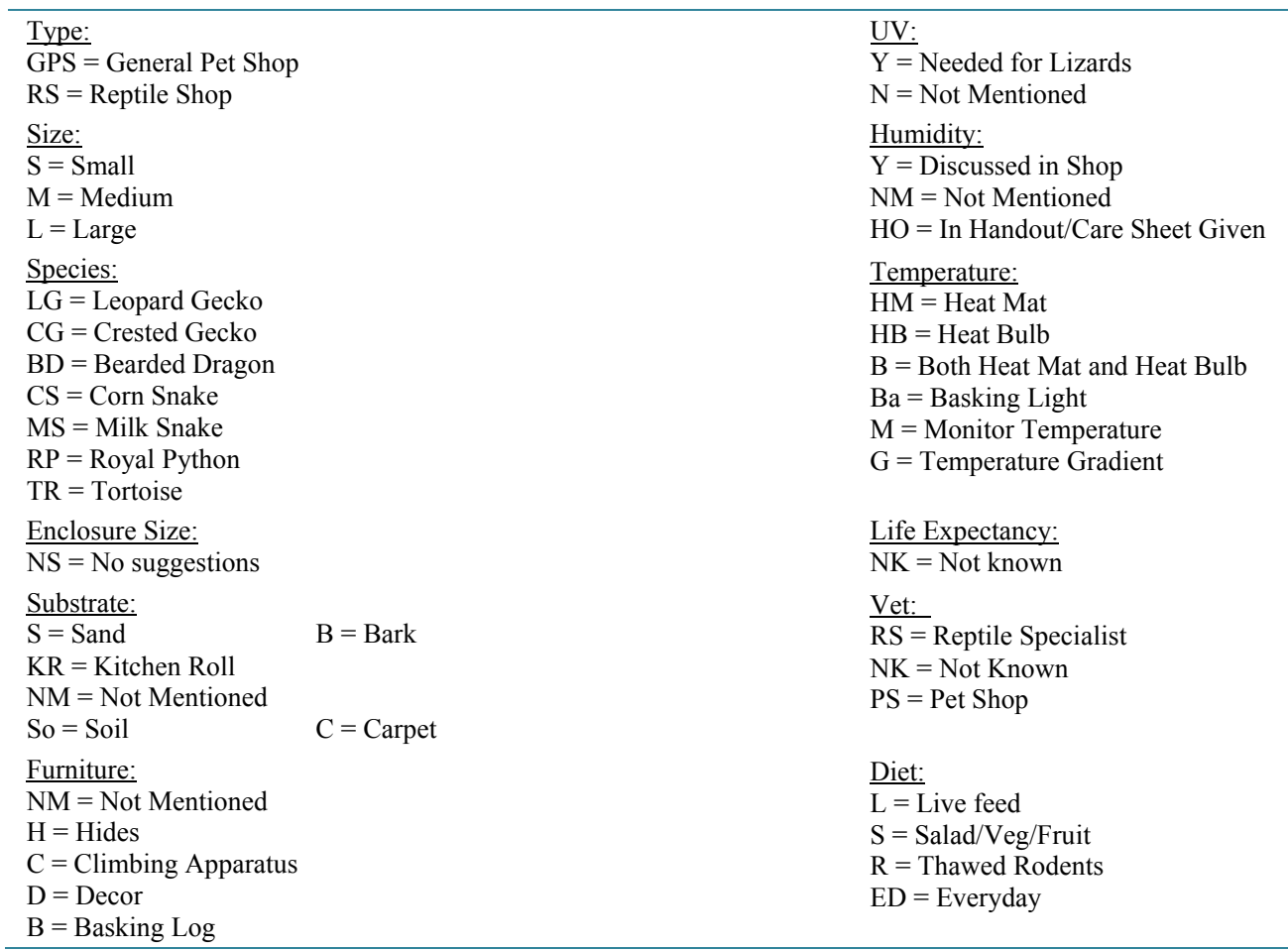


Table 2. Species of reptile recommended by pet stores for novice reptile owners.

\begin{tabular}{cc}
\hline Species Recommended & Percentage of Shops Recommending Species \\
\hline Leopard Gecko & $26.3 \%$ \\
Bearded Dragon & $42.1 \%$ \\
Corn Snake & $17.9 \%$ \\
Crested Gecko & $3.2 \%$ \\
Royal Python & $2.1 \%$ \\
Other & $3.2 \%$ \\
\hline
\end{tabular}

Correct diet is similarly essential for health of captive reptiles and different species require various specific dietary items. All sellers appeared to understand the importance of nutrition as well as the requirements for the species for sale. For example all pet shops selling bearded dragons recommended fruit and vegetables as well as live feed involving mainly crickets. All sellers of leopard geckos recommended live food only. All sellers of corn snakes recommended thawed rodents of varying sizes and at varying intervals depending on the age of the snake. Only $11 \%$ advised not to handle the snake after feeding. Again only a small number (12\%) of the shops visited advised on some sort of vitamin/calcium supplementation.

Concerning signs of health and disease, $88 \%$ of pet shops visited were able to communicate some information regarding what signs to look for concerning an unhealthy reptile. Only $12 \%$ were able to recommend a specific veterinary practice which would be able to provide care for reptiles. $20 \%$ of shops did not know what to recommend while $10 \%$ advised that if there were ever any signs of illness to bring the reptile back to the shop for health care.

When asked how long a reptile purchased could be expected to live stores gave a variety of answers. The life expectancy of a captive corn snake can be up to 20 - 25 years [9] although in captivity they generally life much shorter lives, often only up to 10 years. $75 \%$ of pet shops selling corn snakes advised owners that they would live for longer than this, averaging 15 - 20 years. A captive bearded dragon would be expected to live for $5-10$ years $[10] .50 \%$ of the shops gave advice within this range, whilst the other half gave an average of 15 or more years. Finally the expected life expectancy of the leopard gecko is over 20 years [11]. $68 \%$ of stores advised a much lower life expectancy, averaging $10-15$ years.

\section{Discussions}

Many people to keeping reptiles at first have no concept of the needs of their charges either theoretically through an understanding of the poikilothermic physiology, the nutritional prerequisites and the behavioural requirements of the animal or practically in grasping the technicalities of proving an appropriate captive environment for their new pets. The Animal Welfare Act (2006) requires not only that owners prevent harm occurring to animals under their care, but also that they take actions to promote the individual's health and welfare. For this it is clearly necessary for owners to understand how to provide this for the animals they purchase, and the government provides guides for welfare of a number of companion animal species and for captive primates but not for reptiles. While the internet can provide valuable information it can also be confusing and contradictory. The shop or store from which the reptile is purchased should, however, be an ideal portal for provision of relevant and appropriate information. Here we sought to assess whether the information both verbal and written available in 50 stores selling reptiles was appropriate and accurate.

One problem with such an assessment is defining what a correct answer to many of these questions is. Perhaps we can take a few specific examples to illustrate the difficulty. Asking what substrate should be used for a leopard gecko may yield the response that paper towel is the best material. Sand, woodchips or small pebbles can be ingested and cause gastrointestinal impactions, especially in juveniles. Reptile carpet may be recommended by some but toes can be trapped in the material and soil, while nature, can be a source of pathogenic micro-organisms. Yet owners who wish to create a naturalistic environment may well wish to use bark chippings or sand. This shows the tension between what is the best for the animal from a veterinary perspective and from a reptile hobby owner's viewpoint; this difference potentially explains the variation in recommendation between 
different stores.

On the other hand, there are features that should be universally accepted. Providing an enclosure that allows the animal to fulfil its natural behaviours is essential to promote welfare and thus optimising health [12] [13]. To give one example, arboreal snakes are not only physiologically and anatomically tailored to climbing activity [14] but also behaviourally adapted; it is essential that branches or similar structures are provided to allow such behaviour [15]. Indeed all stores with arboreal reptiles recommended cage furniture which allows climbing. Similarly provision of shelter is essential for any reptile, indeed for every animal of whatever species and $70 \%$ of stores recommended provision of a shelter. Lack of shelter increased plasma cortisol levels in one report on captive viperid snakes when a hear source was also lacking [16].

Such a finding highlights the fact that for reptiles, as poikilothermic species, maintaining a correct temperature is a key feature of optimal husbandry. In fact the key requirement is a temperature gradient to allow reptiles to vary their body temperature at different times of day [17], before and after meals [18], as behavioural pyrexia following bacterial infection [19] or even behavioural hypothermia in periods of exhaustion [20]. So while all stores recommended a source of heat it is concerning that only $8 \%$ mentioned the need for a thermal gradient. Different species may have markedly different optimal temperature zones [21]. Even sympatric species can differ markedly in their thermal environmental requiements [22]. These variations often linked to differences in behaviour in the wild [23].

Given all these factors, it is vital that any newly purchased reptile is given the correct environmental temperature range from the very start of its life with a new owner. Incorrect provision of a thermal source can have fatal consequences from long-term metabolic, digestive and immune malfunction inn hypothermia to more catastrophic conditions such as thermal burns from incorrectly positioned or monitored heat sources [5] [24]. Before purchase of a reptile it is recommended that the owner should undertake research into the temperature requirements of the particular species to be obtained. Is the species predominantly a heliotherm, obtaining heat from basking or a thigmotherm where the animal absorbs most heat from its immediate surroundings? Temperature should be monitored at the cool end of the vivarium so as to ensure it is neither overheating during the day nor dropping too low at night. Heat mats and heat rocks are discouraged by any as they often result in thermal burns and provide only a focal area of heat. No heat sources should be accessible to contact with the reptile and should be guarded.

Humidity is a vital but often neglected component of reptile husbandry. Incorrectly low humidity can result in severe consequences, often in relation to inadequate or incomplete ecdysis [25]. In contrast too high humidity can contribute to the growth of bacterial or fungal pathogens [26]. In natural environments, most small reptiles will spend much of their time in burrows or beneath rocks or leaf litter etc. where humidity is higher and air convection is lower than what is generally perceived. It has been recommended that relative humidity in captivity should be kept at $70 \%$ for all species, with some such as chameleons requiring much higher humid conditions [27].

Many species of reptile require ultraviolet light for cutaneous synthesis of previtamin D3 and the maintenance of active vitamin D in blood [28]. Some reptiles have been raised or maintained successfully without UV light but with dietary supplementation of vitamin D3, and generally ultraviolet light is to be recommended. In order to ensure adequate UV availability, regular changing of the bulbs should be undertaken. While a majority of stores recommended purchase of an ultraviolet light but none gave detailed information about the reasons behind the need for such illumination or how regularly bulbs needed to be changed. More information is needed in this area.

As with all other animals, a balanced diet must be provided which includes all the necessary nutrients for growth and development. This must be of the appropriate quantity, quality and composition for the species, sex, age and season. Malnourishment problems regularly encountered include obesity as a consequence of overfeeding given the low metabolism of reptiles, as well as unawareness of the toxicity of certain substances such as spinach which is high in oxalic acid which can result in circulation disorders. Therefore it is vital that before purchase, a thorough understanding is gained of how to provide a diet that is both attractive and accepted, as well as providing adequate nutrition. For example, most lizards and snakes are sight feeders, whilst skinks and geckos are frugivores, being attracted to sweet nectar-rich fruit. It is important to feed live crickets appropriately, for example with a calcium source which will then be beneficial to the reptile. Other supplementation of vitamins and minerals must be carefully conducted to avoid vitamin toxicities and $\mathrm{Ca}: \mathrm{P}$ imbalance. While a majority of stores gave appropriate advice on feeding the species they offered for sale, further information especially re- 
garding the underlying reasons for the nutrition to be provided would always be valuable.

As noted earlier the most common causes of decreased life expectancy and illness in reptiles are often related to poor husbandry. It is of vital importance that keepers of reptiles are aware of the problems that may be encountered and where to take their animal for veterinary attention. A mere $8 \%$ of stores were able to give some advice regarding signs of ill health and only $12 \%$ were in the position to recommend a local veterinary practice with an interest or expertise in reptile care. It could therefore be recommended that veterinary practices which do provide reptile care should ensure contact with respective pet shops in their area to optimise the opportunity for new owners of reptiles to be directed in their direction.

\section{References}

[1] Pet Food Manufacturers' Association (2013) Pet Population Survey. http://www.pfma.org.uk/pet-population-2015

[2] Cowan, D.F. (1980) Adaptation, Maladaption and Disease. Reproductive Biology and Diseases of Captive Reptiles, Society for the Study of Amphibians and Reptiles, Contributions to Herpetology, 1, 191-196.

[3] Warwick, C., Arena, P., Lindley, S., Jessop, M. and Steedman, C. (2013) Assessing Reptile Welfare Using Behavioural Criteria. In Practice, 35, 123-131. http://dx.doi.org/10.1136/inp.f1197

[4] Ashley, S., Brown, S., Ledford, J., Martin, J., Nash, A.E., Terry, A., Tristan, T. and Warwick, C. (2014) Morbidity and Mortality of Invertebrates, Amphibians, Reptiles, and Mammals at a Major Exotic Companion Animal Wholesaler. Journal of Applied Animal Welfare Science, 17, 308-321. http://dx.doi.org/10.1080/10888705.2014.918511

[5] de Vosjoli, P. (1999) Designing Environments for Captive Amphibians and Reptiles. Veterinary Clinics of North America: Exotic Animal Practice, 2, 43-68.

[6] Hernandez-Divers, S.J. (2001) Clinical Aspects of Reptile Behaviour. Veterinary Clinics of North America: Exotic Animal Practice, 4, 599-612.

[7] RSPCA (2002) Far from Home: Guidelines on Care of Reptiles in Captivity. RSPCA, Horsham.

[8] State of New South Wales (2013) State of NSW and Office of Environment and Heritage. Code of Practice for the Private Keeping of Reptiles. http://www.environment.nsw.gov.au/wildlifelicences/ReptileCode.htm

[9] Robert, K.A., Brunet-Rossinni, A. and Bronikowski, A.M. (2007) Testing the "Free Radical Theory of Aging" Hypothesis: Physiological Differences in Long-Lived and Short-Lived Colubrid Snakes. Aging Cell, 6, 395-404. http://dx.doi.org/10.1111/j.1474-9726.2007.00287.x

[10] Paré, J.A. and Lentini, A.M. (2010) Reptile Geriatrics. Veterinary Clinics of North America: Exotic Animal Practice, 13, 15-25. http://dx.doi.org/10.1016/j.cvex.2009.09.003

[11] Bannock, C.A., Whitasker, A.H. and Hickling, G.J. (1999) Extreme Longevity of the Common Gecko on Motunau Island, Canterbury, New Zealand. New Zealand Journal of Ecology, 23, 101-103.

[12] Meredith, A. and Johnson-Delaney, C. (2010) BSAVA Manual of Exotic Pets. 5th Edition, BSAVA, Shurdington.

[13] Pough, F.H. (1991) Recommendations for the Care of Amphibians and Reptiles in Academic Institutions. ILAR Journal, 33, S5-S21. http://dx.doi.org/10.1093/ilar.33.4.s1

[14] Pizzatto, L., Almeida-Santos, S.M. and Shine, R. (2007) Life-History Adaptations to Arboreality in Snakes. Ecology, 88, 359-366. http://dx.doi.org/10.1890/0012-9658(2007)88[359:LATAIS]2.0.CO;2

[15] Mason, R.T., Hoyt Jr., R.F., Pannel, L.K., Wellner, E.F. and Demeter, B. (1981) Cage Design and Configuration for Arborealreptiles. Laboratory Animal Science, 41, 84-86.

[16] Bonnet, X., Fizesan, A. and Michel, C.L. (2013) Shelter Availability, Stress Level and Digestive Performance in the Aspic Viper. Journal of Experimental Biology, 216, 815-822. http://dx.doi.org/10.1242/jeb.078501

[17] Innocenti, A., Minutini, L. and Foà, A. (1993) The Pineal and Circadian Rhythms of Temperature Selection and Locomotion in Lizards. Physiology and Behaviour, 53, 911-915. http://dx.doi.org/10.1016/0031-9384(93)90268-K

[18] Tsai, T.S., Lee, H.J. and Tu, M.C. (2009) Bioenergeticmodeling Reveals That Chinese Green Tree Vipers Select Postprandial Temperatures in Laboratory Thermal Gradients That Maximize Net Energy Intake. Comparative Biochemistry and Physiology A: Molecular Integrative Physiology, 154, 394-400. http://dx.doi.org/10.1016/j.cbpa.2009.07.011

[19] Merchant, M., Williams, S., Trosclair III, P.L., Elsey, R.M. and Mills, K. (2007) Febrile Response to Infection in the American Alligator (Alligator mississippiensis). Comparative Biochemistry and Physiology A: Molecular Integrative Physiology, 148, 921-925. http://dx.doi.org/10.1016/j.cbpa.2007.09.016

[20] Wagner, E.L., Scholnick, D.A. and Gleeson, T.T. (1999) The Roles of Acidosis and Lactate in the Behavioral Hypothermia of Exhausted Lizards. Journal of Experimental Biology, 202, 325-331.

[21] Warwick, C., Fyre, F.L. and Murphy, J.B. (1995) Health and Welfare of Captive Reptiles. Chapman and Hall, London. 
http://dx.doi.org/10.1007/978-94-011-1222-2

[22] Lelièvre, H., Le Hénanff, M., Blouin-Demers, G., Naulleau, G. and Lourdais, O. (2010) Thermal Strategies and Energetics in Two Sympatric Colubrid Snakes with Contrasted Exposure. Journal of Comparative Physiology B, 180, 415-425. http://dx.doi.org/10.1007/s00360-009-0423-8

[23] Daly, B.G., Dickman, C.R. and Crowther, M.S. (2008) Causes of Habitat Divergence in Two Species of Agamid Lizards in Arid Central Australia. Ecology, 89, 65-76. http://dx.doi.org/10.1890/06-1991.1

[24] Merck, S. and Corp, D. (2010) Selecting a Reptile. Merck and Co., Kenilworth.

[25] Jacobson, E.R. (1977) Histology, Endocrinology and Husbandry of Ecdysis in Snakes. Veterinary Medicine, Small Animal Clinics, 72, 275-280.

[26] Maas III, A.K. (2013) Vesicular, Ulcerative, and Necrotic Dermatitis of Reptiles. Veterinary Clinics of North America: Exotic Animal Practice, 16, 737-755. http://dx.doi.org/10.1016/j.cvex.2013.05.007

[27] Zwart, P. (2001) Pathophysiology: Assessment of the Husbandry Problems of Reptiles on the Basis of Pathophysiological Findings: A Review. Veterinary Quarterly, 23, 14-21. http://dx.doi.org/10.1080/01652176.2001.9695103

[28] Bouillon, R. and Suda, T. (2014) Vitamin D: Calcium and Bone Homeostasis during Evolution. Bonekey Reports, 8, 1-10. http://dx.doi.org/10.1038/bonekey.2013.214 